This item was submitted to Loughborough's Institutional Repository (https://dspace.lboro.ac.uk/) by the author and is made available under the following Creative Commons Licence conditions.

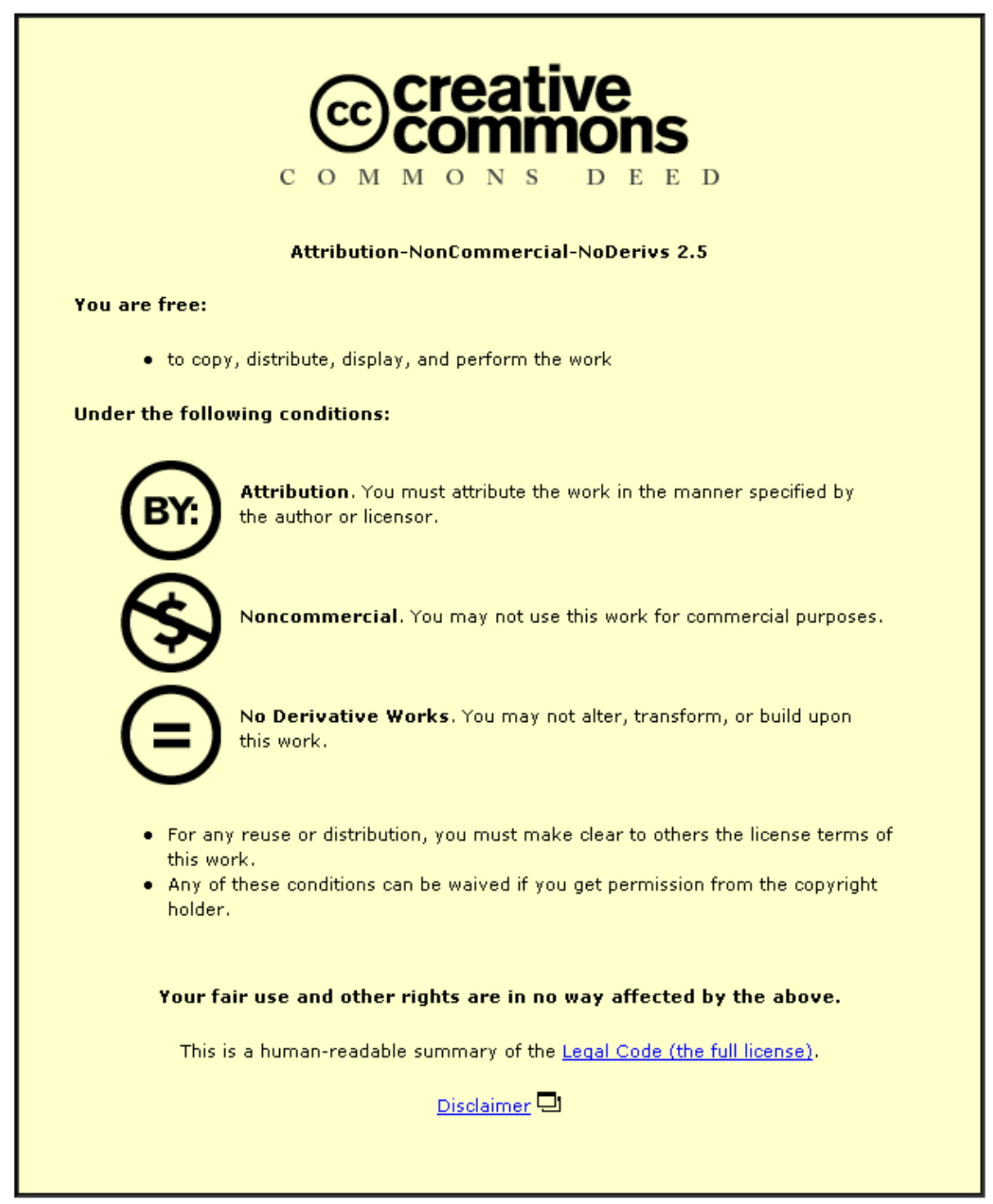

For the full text of this licence, please go to: http://creativecommons.org/licenses/by-nc-nd/2.5/ 


\title{
The Effect of Co-planarity Variation on Anisotropic Conductive Adhesive Assemblies
}

\author{
Guangbin Dou, David C. Whalley and Changqing Liu \\ Wolfson School of Mechanical and Manufacturing Engineering, Loughborough University, Leicestershire LE11 3TU, \\ United Kingdom. \\ Email: gb.dou@lboro.ac.uk; D.C.Whalley@lboro.ac.uk; C.Liu@lboro.ac.uk; Phone: +44(0)1509227677.
}

\begin{abstract}
Anisotropic Conductive Adhesives (ACAs) consist of a polymer adhesive matrix containing fine conductive particles dispersed either randomly, or more rarely in an ordered way. The primary objective of this experimental research was to understand the effects of a non-uniform bond thickness due to non co-planarity of the component or substrate terminations in ACA assemblies. This has been achieved through measurements of the conductivity variations of ACA joints in a number of ACA assemblies, where the component bump plane and substrate plane were deliberately held in different degrees of relative rotation from parallel during adhesive cure. Measurements of the joint resistances versus rotational angle, for a constant bonding force, were made for 10 levels of rotation of the chips relative to the substrates. The results showed that the resistances of the joints in the assemblies exhibited three distinct types of behaviour: stable joint resistances; gradually increasing resistances and unstable resistances. In conclusion, it is shown that ACA joints are very sensitive to the uniformity of the bond thickness, as the larger the rotations were, the lower and less uniform the joint conductivities were, however, the joints were uniform if the rotation angles were controlled within certain limits.
\end{abstract}

\section{Introduction}

ACAs are conceptually a simple method for achieving high density interconnection of electronic components. They offer other potential advantages in terms of low assembly temperature, being lead free, reduced package size, and compatibility with metallizations unsuitable for use with soldering processes $[1,2]$. Whilst unlikely to become a mainstream alternative to soldering for PCB assembly they are well suited to a number of niche applications, such as hybrid circuit assembly, chip on board/flex and LCD assembly. ACAs are therefore experiencing a significant growth in use, although some aspects of their performance, particularly in terms of yield and reliability, are not fully understood. The electronics industry is therefore very interested in the joint reliability of ACA based assemblies. The effect of poor planarity on the ACA joint reliability remains one of the most important issues to be understood before ACAs can be used in really high volumes for low cost applications [2].

There are many factors that affect the yield and performance of electronics packaging. Bonding parameters are generally decisive factors during bonding processes as far as final assembly quality and reliability are concerned. They are even more important in ACA packaging due to the complex mechanical, rheological and chemical properties of ACA materials. Beside bonding parameters, bonding tolerances resulting from bonding process variations or component dimensional variations are other critical factors, which may be difficult to control.

On the one hand, bonding force, bonding temperature and bonding time are the main process parameters in an ACA assembly process. The bonding force affects the compression of the adhesive layer, therefore determining the particle deformation degree, and consequently the contact area between the particles and the component pads [3-5]. Moreover, determining the optimum bonding force is important to achieving a reliable assembly, because the deformation of the conducting particles affects the reliability of the ACA joints $[6,7]$. Bonding temperature and time are other critical parameters for the ACA joint reliability, since they affect the degree of curing of the adhesive resin $[8,9]$. For a given bonding temperature and bonding time, the bonding force influences the final distribution of conducting particles in the ACA assembly $[10,11]$. Therefore, establishing the optimum parameters for ACA bonding will contribute to achieving high quality and reliable electronics assemblies. Recent computational modelling studies of the ACA assembly process have led to a better understanding of the assembly process and facilitate establishment of design rules for different applications [12,13].

On the other hand, beside the parameters mentioned above, ACA bonding tolerances significantly affect ACA assemblies. Generally, there are two kinds of bonding tolerance in ACA packaging, in plane misalignment between the chip and the substrate, which may be translational or rotational, and co-planarity variations between the joint planes. Experimental research on the in plane misalignment of components in ACA assemblies had been carried out in previous research [14]. However, research into the effects of co-planarity variations has been limited.

Misalignment can be caused by chip placement errors, uneven assembly pressure, bump height variation and lack of flatness of the bump pads, and non-uniformity of the ACA thickness [14]. The joint resistances are also very sensitive to the alignment of chip bumps and substrate pads [14]. Bad coplanarity in ACA packaging can be caused by rotational chip misalignment, distorted components and uneven height pads.

In ACA interconnections, the conductive particles provide current paths between the chip bumps and substrate pads through mechanically deformed interfaces. The ACA assembly process is very different from soldering, since the adhesive does not generate surface tension forces to drive the self-alignment process that allows misplaced chips to be pulled into the correct position relative to the substrate electrodes in soldering. Therefore, it is possible to investigate the effect of co-planarity variation on conduction in ACA assemblies by rotating the chip plane relative to the substrate plane, and then thermally locking/curing these rotations in 
place. The purpose of this study was to understand the possible effects of a non-uniform bond thickness, due to non co-planarity of the component or substrate terminations, on the joint resistances and reliability in ACA assemblies.

\section{Materials and Methods}

\subsection{Materials}

\subsubsection{ACA}

ACAs are designed to achieve fine pitch electronics assembly, providing electrical paths as well as mechanical connections between the conductive pads on electronic components and substrates. There are two forms of ACA: films, which are often referred to as ACFs; and pastes, refereed to as ACPs. There are also two types of adhesive resins used, thermosetting and thermoplastic, and three kinds of particles: rigid or solid metal particles; compliant or polymer cored particles; and solder particles. [15]. Figure 1 shows all these options.

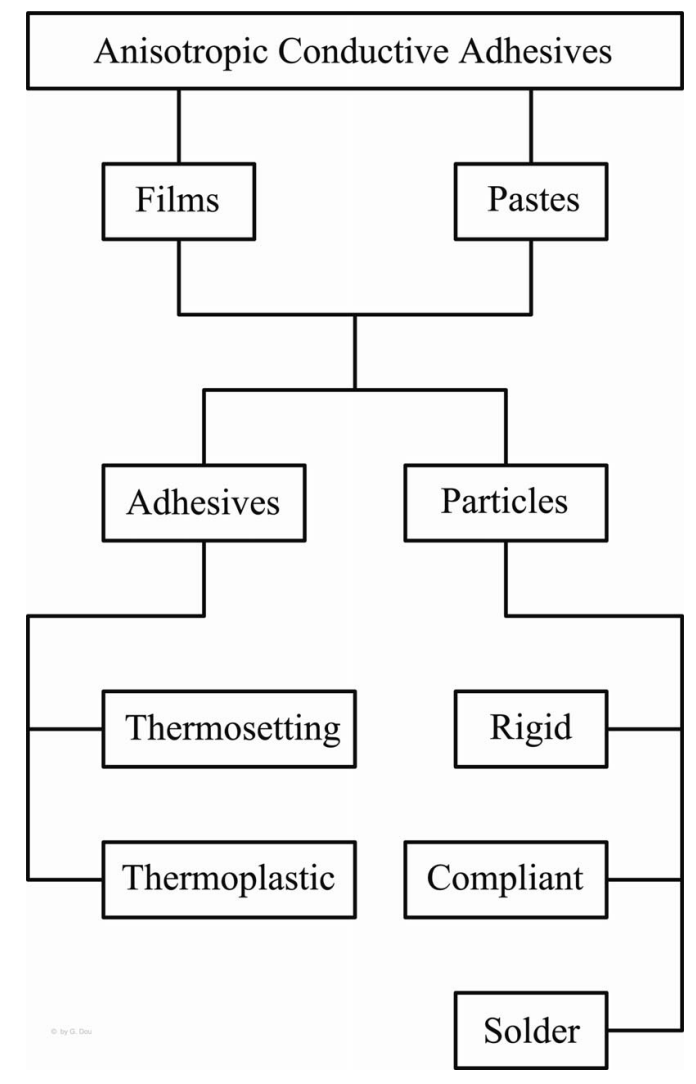

Fig. 1. ACA types and their typical compositions[15]

ACA particles are generally dispersed randomly in the polymer at a low density, however, ACAs have been developed where the particles are uniformly separated in the same non-conductive plane [16]. These two types of materials are referred to as random ACAs, and ordered ACAs respectively.

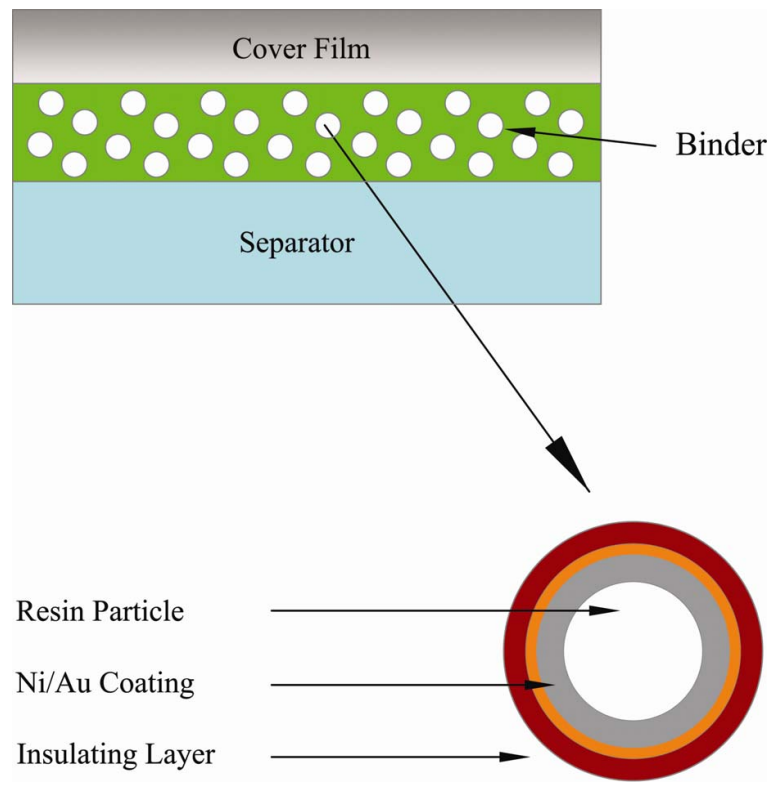

Fig. 2. ACA configuration

A commercial ACF, Sony 1058E, was used in this research, which was specially designed for chip-on-flex (COF) packaging, and therefore has the capability of providing reliable fine pitch assembly. The adhesive is supplied with a cover film and separator on opposite sides of the film, as shown in Figure 2. The material is thermosetting, with compliant conductive filler particles. The conductor particles are nickel and gold coated resin balls, as the cross section of a particle illustrates in figure 2. The particles, 3.5 $u \mathrm{~m}$ in diameter, are randomly dispersed in the ACF layer, which is $35 u \mathrm{~m}$ in thickness, with a density of 3.5 million $/ \mathrm{mm}^{3}$. This resulted in a final particle density on the pads of about $5 \times 10^{3} / \mathrm{mm}^{2}$.

\subsubsection{Chips and Substrates}

Aluminium metallised $\mathrm{Si}$ chips with $\mathrm{Ni} / \mathrm{Au}$ coated $\mathrm{Cu}$ bumps and Flexible Printed Circuits (FPCs) with $\mathrm{Ni} / \mathrm{Au}$ over copper pads were used in this experiment.

The chip size was $11 \mathrm{~mm} \times 3 \mathrm{~mm}$ and $0.5 \mathrm{~mm}$ in thickness, and the bump height was $4 \mu \mathrm{m}$ including the $N i$ and $A u$ layers. The chips used in this research were bumped along their four sides, as shown in Figure 3, but only the long sides were interconnected such that electrical resistance measurements could be made. There were 150 bumps in each long side of the chip, the bump size was $50 \mu m \times 70 \mu m \times 5 \mu m$, and the bump pitch was $75 \mu \mathrm{m}$. The distance between the long rows of the bumps was $2.53 \mathrm{~mm}$.

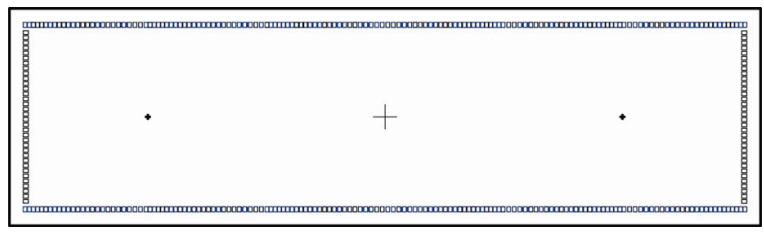

Fig. 3. Chip pattern 
Upilex FPC substrates, $25 u \mathrm{~m}$ in thicknesses, with the pattern shown in Figure 4, were used in these experiments. The substrate pad height was $14.5 u$ m including the $\mathrm{Cu}, \mathrm{Ni}$ and $A u$ layers.

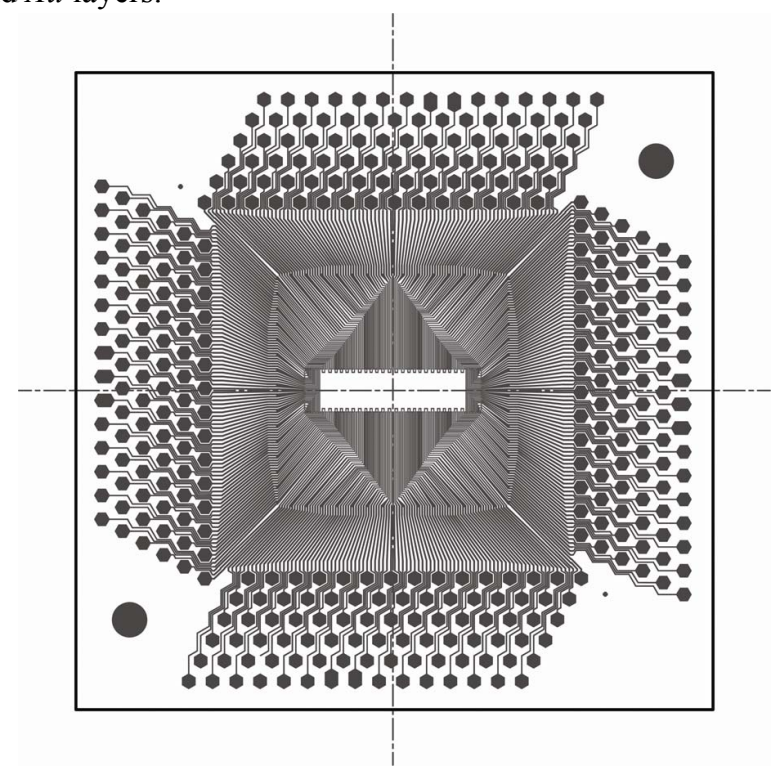

Fig. 4. Substrate pattern

The joint resistance measurements were achieved by using a four wire method on one joint in each group of five, using the design illustrated in Figure 5, which schematically shows the substrate pads and chip bumps, including the initial $A l$ layer [17]. The test current was applied through connections 1 and 5 and voltage sensing was through connections 3 and 4 . This arrangement therefore allowed measurement of the resistance of 30 pads along each long side of the package.

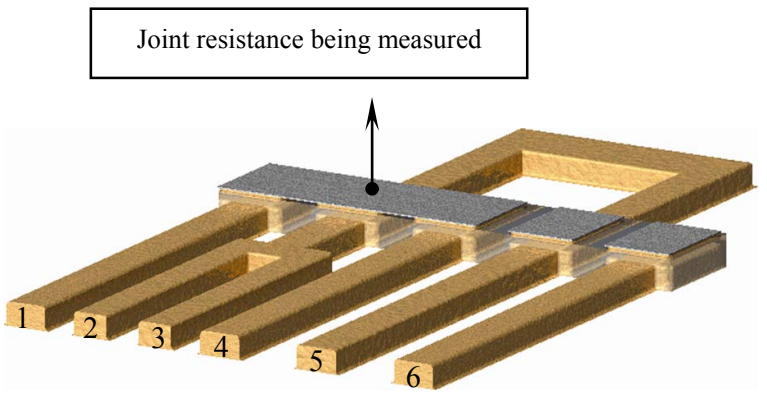

Fig. 5. ACA joints for FPP measurement

\subsection{Methods}

\subsubsection{Experimental Design and Approach}

Co-planarity issues in ACA assembly are complex since there are many factors that can cause poor planarity connections, such as the pads on the components not lying in the same plane due to manufacturing errors/tolerances, the distortion of the components due to the bonding conditions, and incorrect alignment due to bonding machine tolerances.
In this experiment, co-planarity variations were deliberately introduced by rotation of the chips through a range of small angles, as shows in Figure 6.

Basically, there are two possible kinds of chip rotations for a rectangular chip, rotation along the long chip side and rotation along the short chip side, which are referred to as $\alpha-$ rotation and $\beta$-rotation respectively in this study. Co-planarity issues in practical assemblies may result from a mixture of these two rotations, and may also include misalignment problems. This paper concentrates on $\alpha$-rotation issues, whilst research into the effects of $\beta$-rotation will be carried out and reported subsequently.

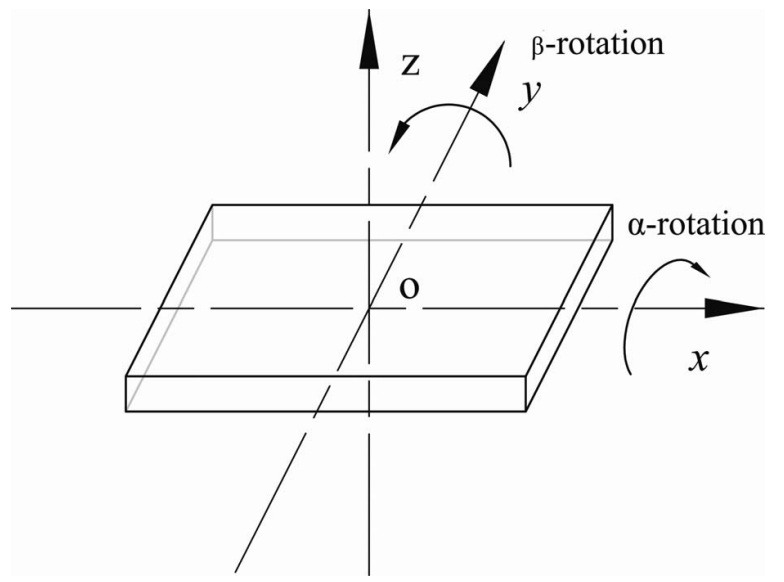

Fig. 6. Chip rotation

In the experiment, all the assemblies were made using a high precision bonding machine, therefore it could be assumed that the joint plane formed by the substrate pad surfaces was a horizontal plane and that the $x y$ plane of the chip, as shown in Figure 6, was normally parallel to this horizontal plane. Different levels of co-planarity error in the assemblies were then obtained by rotating the chip to different angles and locking this angle in during the final-bonding procedure.

If the chip is rotated around the $x$ axis, one of the long chip sides is lifted up and the other is moved down in relation to the $x y$ plane, as illustrated in the cross section of $\alpha$-rotation in $y z$ plane in Figure 7 . The joints along the lifted up side were therefore less compressed, and those along the lower side were highly compressed. All joints in the same long side of the chip were at the same level.

10 samples were manufactured with $\alpha$-rotations of $\alpha=\operatorname{atan}\left(1 \times 10^{-3}\right), \quad \operatorname{atan}\left(2 \times 10^{-3}\right), \quad \ldots \quad \operatorname{atan}\left(10 \times 10^{-3}\right) . \quad$ The difference in height between the two rows of pads increased by about $1.3 u \mathrm{~m}$ for each level of rotation, which is just over one third of the conductor particle diameter. 


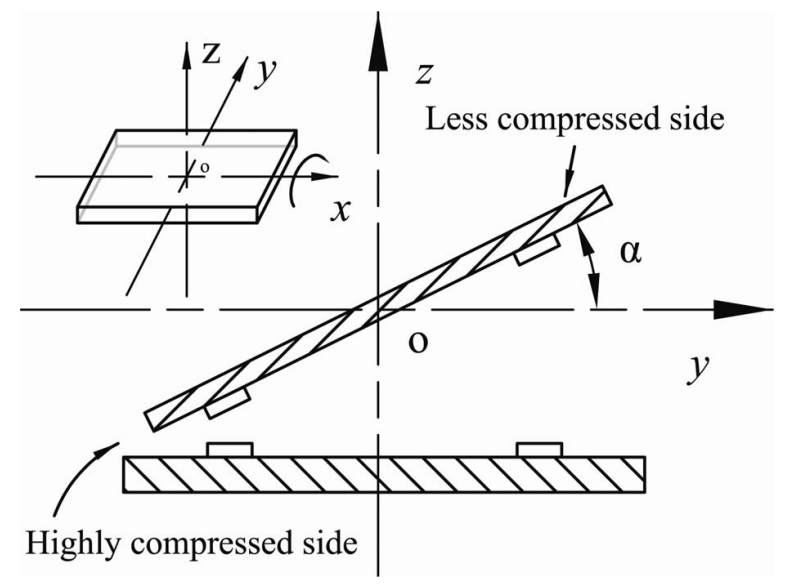

Fig. 7. ACA joints for FPP measurement

\subsubsection{ACA Bonding Process}

ACA assembly requires a dedicated bonding machine due to the fine pitch pads on the components and the specific bonding force and time/temperature requirements. Two flip chip bonding machines, a Karl Suss 9493 Mauren and a Toray (FC1200-2k-\#95) were used for pre-bonding and final bonding respectively. The bonding parameters used are summarized in Table I and were selected according to the ACF specification.

Table I: Bonding Parameters for the ACA Assembly

\begin{tabular}{l|c|c|c}
\hline Process & Temp. $\left({ }^{\circ} \mathrm{C}\right)$ & Pressure (Mpa) & Time (s) \\
\hline Pre-bonding & 80 & 10 & 5 \\
\hline Final bonding & 180 & 100 & 15 \\
\hline
\end{tabular}

Successful assembly relies upon the trapping of conductive particles between the conductive pads of the two parts being connected, followed by the solidification of the adhesive thereby locking in residual stresses to ensure retention of sufficient contact force to create stable and low resistance electrical connections [12]. There are three main steps in the bonding procedure, as shown in Figure 8. Initially, pre-bonding as shown in Figure 8-a is used to laminate the prepared ACF onto the substrate using a low pressure and temperature. Subsequently, the chip is accurately aligned to the pre-bonded substrate using a dedicated machine, as shown in Figure 8-b. The final-bonding is then achieved by applying pressure and heat to the chip, as shown in Figure 8-c, and the assembly process concludes by controlled cooling to room temperature. This experiment was designed mainly to study the effects of the second step, i.e. the alignment process.

\section{Results}

The raw resistance results from the experiment were difficult to interpret, because there are so many factors that can affect ACA joint resistances. ACA joint resistances for the same bonding conditions and same bonding machine, but packaged at different times, can vary significantly due to other uncontrolled factors. The deviation of the joint resistance from the mean/standard value must be within an acceptable range, otherwise the process cannot be used in electronics products. Therefore a quality control concern in the electronics industry is the achievement of consistent joint resistances.

The results shown in this paper are the mean values of the joint resistances for the same level of compression from the same samples, together with results for the standard deviation of the resistances and the numbers exceeding the acceptable level of resistance, $100 \mathrm{~m} \Omega$.

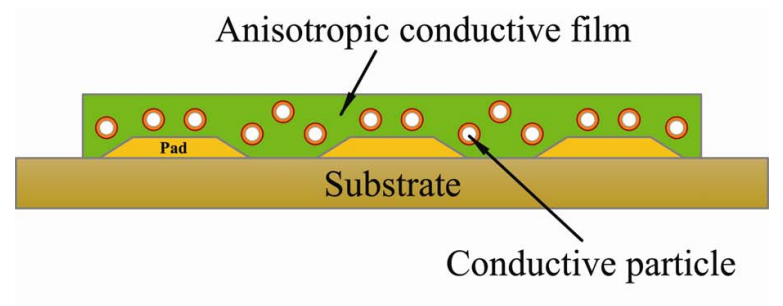

a)
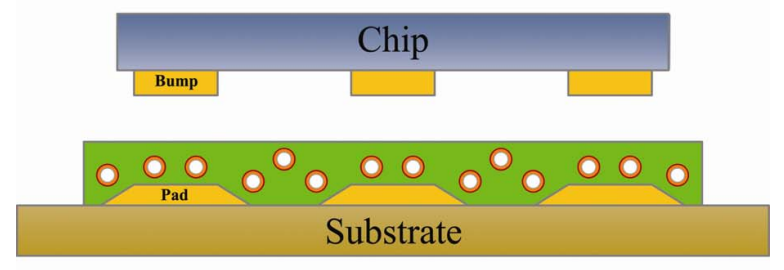

b)

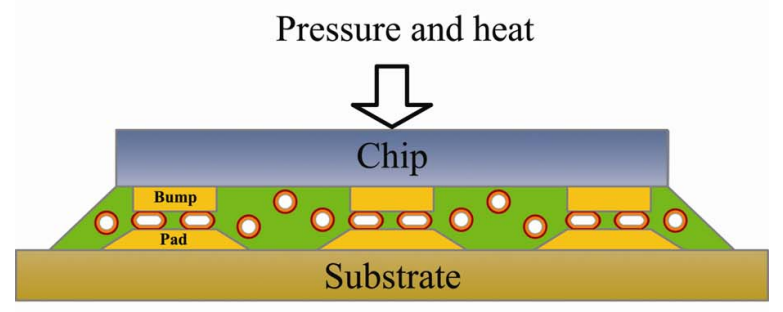

c)

Fig. 8. ACA Bonding Process

\subsection{Less Compressed Side}

The results presented in Figure 9 are the mean values for the less compressed side of samples with different imposed rotation angles. Each value in the figure is the average (arithmetic mean) of the results for the 30 joint resistance measurements from the less compressed side of a sample, except that for an angle of 0 , which is the mean value of the 120 joints measured on 2 samples without rotation, so it can 
be taken as a standard value. The error bars show the standard deviation of resistance, giving an indication of the consistency/spread of the resistances.

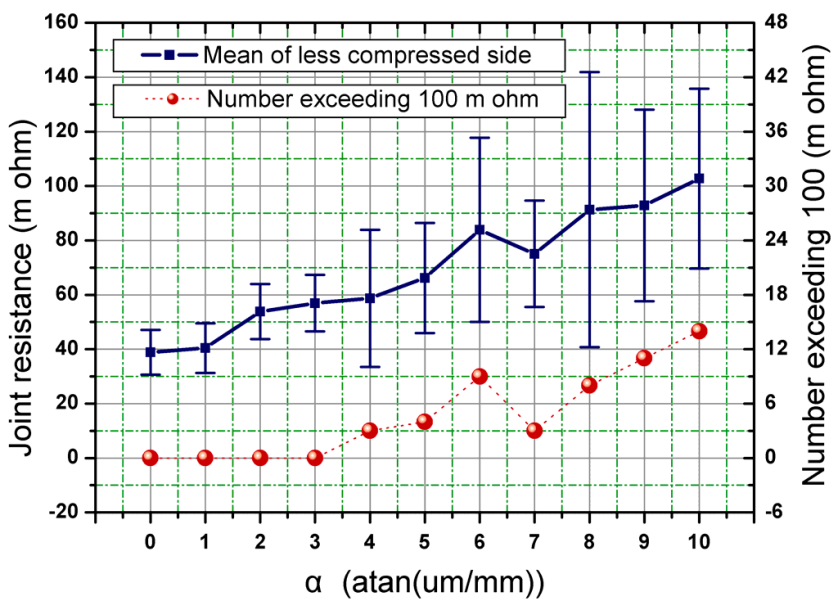

Fig. 9. Resistance results for the less compressed side

It can be seen that the mean joint resistance increased reasonably linearly from about $40 \mathrm{~m} \Omega$ to more than $100 \mathrm{~m} \Omega$ as the rotation angle increased, although the joint resistances did not change significantly for the smallest level of rotation. The spread in resistance values also tended to increase with increasing rotation angle, although not in such a linear way. These increases in resistance may be attributed to the reduced compression of the conductor particles and may also be due to a reduced number of trapped particles caused by the greater flow of ACA resin over the pads during the final bonding, due to the tilted chip forcing all of the resin out of that side of the chip. Flow of the adhesive over the pads has previously been shown to reduce particle density by Mannan et al. [18]. Furthermore, the variation of the mean joint resistances for large rotation angles indicated that the joint yield may be significantly reduced. For instance, the highest joint resistance in sample 8 was more than $240 \mathrm{~m} \Omega$, and some high resistance joints (defined here as more than $1000 \mathrm{~m} \Omega$ ) were found in the less compressed side in some of the samples with larger rotations (samples 8, 9 and 10), i.e. there were 3 high resistance joints in sample 8,1 in sample 9 and 2 in sample 10. The resistance variations in samples $0,1,2$ and 3 were all relatively small, but from sample 4 to sample 10 , the spread increased dramatically, reaching their maximum for sample 8 . The large rotation angle assemblies will definitely cause yield problem because some joint resistances, were too large to be used. It is not clear why the mean joint resistance for sample 7 was smaller than for sample 6 , however it is probably a random effect due to variability in the materials and bonding process.

Figure 9 also shows the numbers of joints in each sample with a resistance exceeding the acceptable threshold of 100 $m \Omega$. The numbers showed that some of the joints in the samples were not acceptable after angle 3, and the larger the angle was, the more joints exceeded the threshold.
To sum up, there were three stages in the less compressed side in this experiment. Stage I, the stable stage, was identified as being from sample 0 to 1 as the mean joint resistances were concerned. It can be seen there was almost no difference in joint resistances within this small amount of small rotation. As for stage II, from sample 2 to 6 , the mean joint resistances increased gradually due to the decreased deformation of the ACA particles as the rotation angle increased step by step. Variation of the mean joint resistances was found from sample 7 to 10 in stage III. In this stage, besides the large resistances resulting from the small deformation of the ACA particles, the conductivity of the mechanical contacts reduced due to the dramatically reducing area of contact between the particles and the pads. The probably lower number of particles trapped between the bumps and the pads, due to the increased ACA matrix flow over the pads as the rotation increased, is another factor that could have contributed to the large and variable joint resistances.

\subsection{Highly Compressed Side}

Compared to the less compressed sides of the same samples, the mean results for the highly compressed side were much more consistent. The results are presented in Figure 10, to the same scale as in Figure 9. All of the mean resistance values were between $40 \mathrm{~m} \Omega$ and $60 \mathrm{~m} \Omega$ and none of the resistances exceeded the acceptable threshold of $100 \mathrm{~m} \Omega$, and the highly compressed side joints were more uniform than those in the less compressed side.

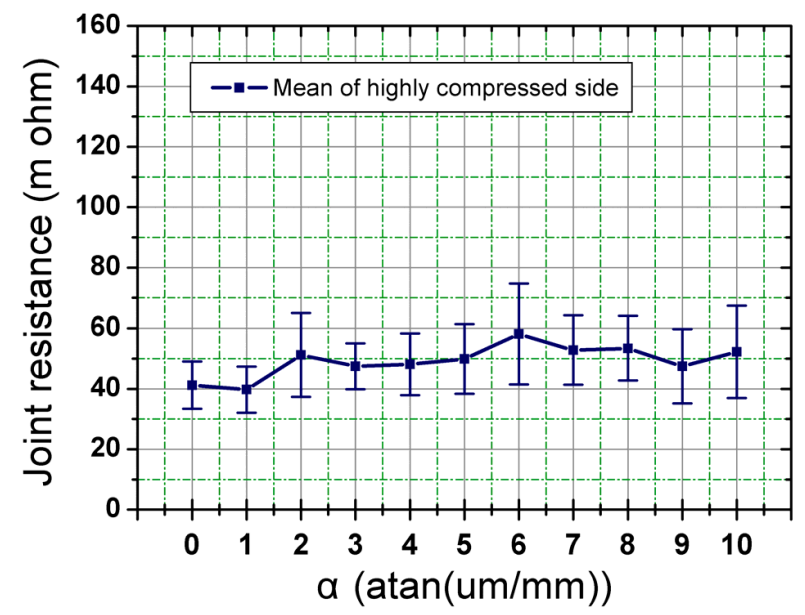

Fig. 10. Results for the highly compressed side

In order to achieve a detailed understanding of the effect of high levels of compression on the ACA assemblies, the results for the highly compressed side were also divided into three stages according to the resistance pattern shown in Figure 9. As for the less compressed side, the three identified stages are:

stage I: sample 0---1;

stage II: sample 2---6;

stage III: sample 7---10. 
Stage I: The mean joint resistances as shown in the figure dropped a little, which is believed to be because the particles trapped between the bumps and the pads in sample 1 were deformed a little more than in sample 0 , increasing the contact area. Stage II: The trapped particles between the bumps and the pads were becoming over compressed and therefore crushed as the rotation increased, therefore the interconnection resistances increased to slightly higher than those in the stage I. The crushed particles resulted in low conductivity, because of the cracks in the particles and also the reduced mechanical contact areas between the particles and the pads. The worst situation in this stage was that most of the particles were crushed, and there were few apparent mechanical contacts between the bumps and the pads. In such cases, the joint resistances may be quite high, some of them were as high as $95.2 \mathrm{~m} \Omega$ in sample 6 , because of the cracks in the particles and the bad contacts between the particles and the pads. Stage III: The ACA particles were crushed deeply in this stage due to the large pressure caused by high levels of compression along the long chip side. Consequently, there was a lot of direct mechanical contact between the bumps and pads, where the situation became similar to that in None Conductive Adhesive (NCA) assembly, however the pieces of crushed resin coated particles may reduce the area of direct mechanical contact between the metal pads, resulting in a worse conductivity than for a NCA.

\section{Conclusions}

This set of trials has generated an understanding of the effects of the level of co-planarity variation on ACA joint resistances. It has been confirmed that poor co-planarity, can result in low conductivity joints in the assemblies.

Three stages of performance were identified from the different samples as their rotation degree were increased, as shown in Figure 11.

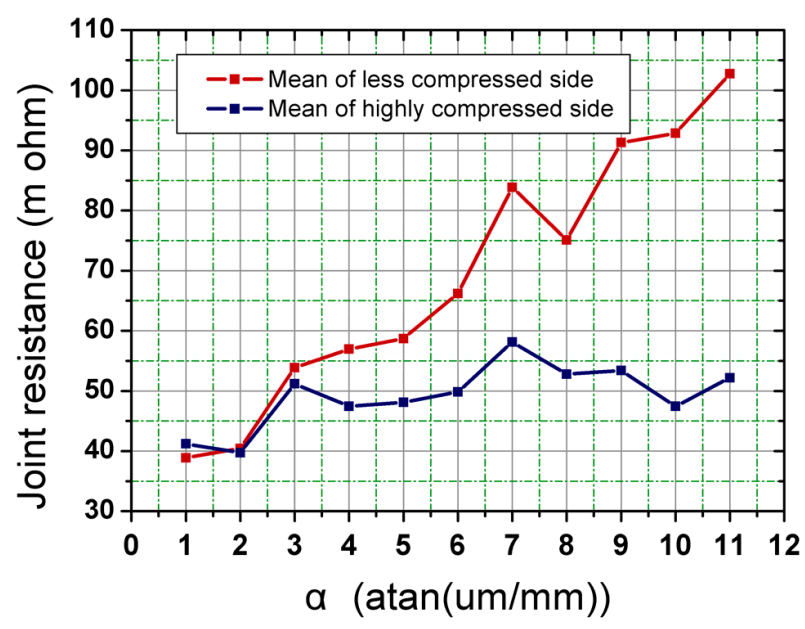

Fig. 11. Mean values of co-planarity rotation

In stage I, low and uniform resistances were found. In stage II, the joint resistances of the less compressed side increased, due to the decreased deformation of the ACA particles. In contrast to the less compressed side, the lower conductivity joints in the highly compressed side resulted from crushed particles and the reduced area of mechanical contact between the particles and the pads. In stage III, highly variable ACA joints were found in the less compressed side, because the ACA particle deformation was dramatically decreased and the mechanical contact between the particles and bumps and pads were very limited. A smaller number of particles trapped in the joints may be another factor that resulted in non-uniform conductivity in the less compressed side.

Further work will be carried on the $\beta$-rotation for comparison with the $\alpha$-rotation results presented here. Research is also being conducted into how the deformation forces on the individual particles are affected by the bonding forces in assemblies with different co-planarities which will be reported in a separate study.

\section{Acknowledgments}

The authors would like to acknowledge Prof. Y. C. Chan from the Department of Electronic Engineering, City University of Hong Kong where Mr. Dou conducted some of the experimental work.

\section{References}

1. Liu, J., Conductive Adhesive for Electronics Packaging, 1999, Electrochemical Publications Ltd., Port Erin, Isle of Man, British Isles

2. Liu, J.H. "Life Time Prediction of Anisotropic Conductive Adhesive Joints During Temperature Cycling for Electronics Interconnect," Proc $1^{\text {st }}$ International IEEE Conference on Polymers and Adhesives in Microelectronics and Photonics Conf, Potsdam, Germany, October. 2001, pp. 209-212.

3. Yeung, N.H., Chan Y.C., and Tan C.W., "Effect of Bonding Force on the Conducting Particle with Different Sizes", Journal of Electronic Packaging, 125(4), 2003, pp. 624-629.

4. Dou, G.B., Chan Y.C., and Liu J., "Electrical Conductive Characteristics of Anisotropic Conductive Adhesive Particles", Journal of Electronic Packaging, 125(4), 2003, pp. 609-616.

5. Maattanen, J., "Contact resistance of Metal-coated Polymer Particles used in Anisotropically Conductive Adhesives", Soldering \& Surface Mount Technology, 15(1), 2003, pp. 12-15.

6. Chan, Y.C. and Luk D.Y., "Effects of Bonding Parameters on the Reliability Performance of Anisotropic Conductive Adhesive Interconnects for flip-chip-on- Flex Packages Assembly II. Different Bonding Pressure", Microelectronics Reliability, 42(8), 2002, pp. 1195-1204.

7. Chan, Y.C. and Luk D.Y., "Effects of Bonding Parameters on the Reliability Performance of Anisotropic Conductive Adhesive Interconnects for flip-chip-on-flex Packages Assembly I. Different Bonding Pressure", Microelectronics Reliability, 42(8), 2002, pp. 1185-1194.

8. Seppala, A. and Ristolainen E., "Study of Adhesive Flip Chip Bonding Process and Failure Mechanisms of ACA Joints", Microelectronics Reliability, 44(4), 2004, pp. 639648. 
9. Seppala, A., Aalto K., and Ristolainen E., "Reducing Bonding Cycle Time of Adhesive Flip Chip Process", Soldering \& Surface Mount Technology, 15(1), 2003, pp. 16-20.

10. Ogunjimi, A.O., Mannan, S. H., Whalley, D. C., Williams, D. J., "The Assembly Process for Anisotropic Conductive Joints --- Some New Experimental and Theoretical Results", Journal of Electronics Manufacturing, 5(4), 1995, pp. 263-271.

11. Dudek, R., Meinel, S., Schubert, A., Michel, B., Dorfmuller, L., P. M. Knoll, Baumbach, J., "Flow Characterization and Thermo-mechanical Response of Anisotropic Conductive Films", IEEE Transactions on Components and Packaging Technologies, 22(2), 1999, pp. 177-185.

12. Whalley, D.C., Glinsky, G., Bailey, C., Liu, J. H., "Computational Modelling of the Anisotropic Conductive Adhesive Assembly Process", Proc $3^{\text {rd }}$ International Symposium on Electronics Materials and Packaging, Jeju, Korea, November 2001, pp393-398.

13.Whalley, D.C., Kristiansen, H. and Liu. J., "Characterisation of Anisotropic Conductive Adhesive Compression During the Assembly Process", Advances in Electronic Packaging, Proc of the 2003 ASME InterPack conference, Hawaii, Vol. 1, pp. 183-189.

14. Fan, S.H. and Chan Y.C., "Effect of Misalignment on the Electrical Characteristics of Anisotropic Conductive Adhesive Joints", Proc 52 $2^{\text {nd }}$ IEEE Electronic Components \& Technology Conf, San Diego, CA. May. 2002, pp. 1605-1602.

15. Dou, G.B., Whalley D.C., Liu C., "Electrical Conductive Charateristics of ACA Bonding: A Review of the Literature, Current Challeges and Future Prospects", Proc $6^{\text {th }}$ IEEE Conference on High Density Microsystem Design and Packaging and Component Failure Analysis (HDP'04), Shanghai, China, June. 2004, pp. 264-276.

16. Holloway, M. "Novel Anisotropically Conductive Film for Area Array Packaging", Advances in Electronic Packaging, Proc of the 1999 InterPack conf, ASME, Hawaii, June.1999, Vol. 2. pp.1699-1704.

17. Dou G.B., Chan Y.C., Morris J.E. and Whalley D.C., "RLC Effects in Fine Pitch Anisotropic Conductive Film Connections", Soldering \& Surface Mount Technology, 18(1), 2006, pp. 12-15.

18. Mannan S.H., Whalley D.C., Ogunjimi A.O. and Williams D.J., "Assembly of Planar Array Components Using Anisotropic Conducting Adhesives---A Benchmark Study: Part II---Theory", IEEE Transactions on Components, Packaging, and Maunufacturing Technology---Part C, 19(4), 1996, pp. 264-269. 